about ACP and having the conversations), were delivered by a small national group of national trainers. 63 workshops $(907$ participants) were delivered in 2017. These workshops reported a statistically significant increase in clinician confidence to have ACP conversations. The District Health Boards (DHBs) wanted to increase the number of workshops being delivered and to take greater local control of the training.

Method The national ACP team worked with a team of trainers to develop a train-the-trainer course to train local DHB trainers to deliver the L1A workshops. 41 local DHB trainers have been trained. There was concern that delegation of training delivery to local trainers might impact the quality of the workshops. To mitigate against this risk, trainee trainers are required to go through a rigorous 6 step training and accreditation process before being accredited as trainers of the L1A workshop.

Results The preliminary evaluation of the train-the-trainer programme finds that it meets the expectations of trainee trainers and leaves them feeling prepared to deliver the workshops locally. Initial assessment indicates that the increase between pre-and post- workshop confidence scores of participants remains statistically significant.

\section{OP94 STANDARDIZED PATIENTS FOR THE ACP-FACILITATOR QUALIFICATION: ENHANCE YOUR TRAINING}

${ }^{1} \mathrm{~K}$ Götze* ${ }^{2} \mathrm{~S}$ Otten, ${ }^{1} \mathrm{~J}$ In der Schmitten. ${ }^{1}$ Heinrich-Heine University, Düsseldorf, Germany; ${ }^{2}$ CoMed Communication in medical education

10.1136/spcare-2019-ACPICONGRESSABS.93

Summary of workshop In 2017, after delivering some 14 ACPacilitator workshops in 3 years, we redesigned our facilitator workshop to a $72 \mathrm{~h}$-training incl. $24 \mathrm{~h}$ of a standardized patient (SP) - supported role-play training that allows to teach ACP-specific attitudes and skills, with a focus on identifying and adequately responding to emotional barriers.

In an interactive workshop approach, we will first share and discuss the process and lessons of developing SP roles, training the SP, working with the SP-supported facilitator training, caring for SP in the field, and developing a transferrable SP-trainer-trainer system to support ACP-facilitator-qualification at six German-speaking facilitator training sites. Secondly, the participants will work in small groups on developing criteria for meaningful rolescripts and practice writing one. Thirdly, we will present a role-play and thereby demonstrate the interactive training technique that we developed for our workshops.

Learning objectives Think about standard and challenging ACPSituations

Appreciate what SP need in order to do a good job

Write small sequences of a SP role-script

Identify the required steps and depth a SP-training needs to really make a change in teaching complex conversations

Experience interactive SP-Training

Anticipated outcome of the workshop

After the workshop, the participants will ...

... understand the SP-training that needs to be done in order to qualify them for their role in ACP workshops

... be aware of interactive trainer techniques that allow effective learning

... be inspired to include SP-supported training elements in their facilitator training, or enhance existing trainings.

\section{Poster Presentations}

\section{P01 THE NEEDS OF CARING RELATIVES IN RELATION TO ADVANCE CARE PLANNING}

D Drewniak* , I Karzig, T Krones. University of Zurich, Zurich, Switzerland

\subsection{6/spcare-2019-ACPICONGRESSABS.94}

Background Relatives of patients in need of care and of those who are seriously ill assume a variety of tasks. The aim of this study is a systematic review on needs, chances, risks and barriers of care giving relatives regarding advance care planning (ACP).

Methods Qualitative and quantitative studies were identified through Pubmed, EMBASE, PsycINFO und CINAHL searches. In order to take into account that qualitative and quantitative studies were included, data were thematically synthesized.

Results In total 37 studies met inclusion criteria, including 24 quantitative- and 13 qualitative studies. Most studies originated from the USA (46\%). Thematically, the studies can be divided into four different categories: "Attitudes towards ACP" showed that, even though relatives experience some uncertainty about the meaning of ACP, they mostly agree with the necessity of it. "Decision Conflicts" revealed that the level of congruence between relatives and patients is not given in many cases. Regarding the "Effectiveness of ACP" several studies highlighted the importance to consider family dynamics in the ACP process. Different "Barriers for ACP" were found, including a lack of knowledge and awareness about ACP and difficulties regarding the timing of ACP discussions.

Conclusion Although being an important group in the realm of ACP, as relatives are often also surrogate decision makers in case of incapability of decision making, studies on their attitudes and experiences are relatively rare and their knowledge seems quite limited albeit a perceived need for timely and appropriate ACP.

\section{PO2 SERIOUS ILLNESS CONVERSATION GUIDE IMPLEMENTATION AND CUSTOMISATION OF THE GUIDE FOR NEW ZEALAND}

L Manson*, J Goodwin. Health Quality Safety Commission, Wellington, New Zealand

\subsection{6/spcare-2019-ACPICONGRESSABS.95}

Background In New Zealand, clinicians are not routinely taught to have effective and compassionate conversations with consumers about what matters to them nor are they taught to use that information in partnership with consumers to plan and deliver care that matches that. New Zealand District Health Boards recognised the need to enhance the clinical communication skills of their workforce and tasked the Health Quality \& Safety Commission with designing and delivering training to enhance the clinical communication skills of the non-palliative care workforce. As a first step, the Commission has been working with Ariadne Labs to bring the Serious Illness Conversation Guide tool and training to New Zealand. With the differences in the culture of the United States and New Zealand, the Serious Illness Conversation Guide needed to be adapted to the local environment.

Method In August 2018, three codesign workshops were held with 43 consumers and clinicians. At the workshops participants were asked to work together to consider the language 